\title{
A Closed General Solution of the Probability Distribution Function for Three-Dimensional Random Walk Processes*
}

\author{
Chi-hua Hstung, Hsten-chit Hsiung, and Adon A. Gordus \\ Department of Chemistry, The University of Michigan, Ann Arbor, Michigan
}

(Received June 27, 1960)

\begin{abstract}
A closed general solution of the probability distribution function for three-dimensional random walk processes is derived. In addition: (1) For the particular case of equal-length displacements, the exact solution is compared with the Gaussian approximation for $n=3,5$, and 10 steps. (2) The general solution is utilized in calculating the probability distribution of gamma-ray energies resulting in the $\mathrm{Cl}^{35}(n, \gamma) \mathrm{Cl}^{36}$ process. (3) For five unequal steps of fractional length: $0.582,0.135,0.131,0.092$, and 0.060 (which is somewhat characteristic of the fractional energies of gamma rays resulting from neutron capture), the exact solution is compared with a Gaussian, a modified Gaussian, and a five equal-step approximation. (4) There are presented the specific solutions for all possible unequal-length random displacements involving $n=2,3$, and 4 steps.
\end{abstract}

\section{INTRODUCTION}

W $\mathrm{E}$ have been interested in using the random walk model to evaluate the probability distribution of the recoil energy imparted to an atom in a molecule as a result of neutron-capture gamma-ray emission. Cobble and Boyd ${ }^{1}$ have discussed this problem with regard to the $\mathrm{Br}^{81}(n, \gamma) \mathrm{Br}^{82}$ process. In the case of cascade gamma emission there exists (in many cases) a finite probability that the atom activated by neutron absorption receives a relatively small recoil energy and thus fails to dissociate from the parent molecule. ${ }^{2}$ If neutron-capture gamma-ray data are available for the process of interest, and if the gamma-ray emission is spherically symmetrical, then the probability distribution of energies can be calculated in terms of the random walk model.

If the $i$ th step is of length $l_{i}$ and in a random direction in space, then, according to Rayleigh, ${ }^{3,4}$ the probability distribution function will be

$W_{n}(R)$

$=\left(2 \pi^{2} R \prod_{i=1}^{n} l_{i}\right)^{-1} \int_{0}^{\infty}\left\{\left[\prod_{i=1}^{n} \sin \left(l_{i} \rho\right)\right] \sin (R \rho) / \rho^{n-1}\right\} d \rho$,

where $R$ is the vector sum of $n$ random displacements $l_{i}(i=1$ to $n), \rho$ is a dummy variable of spatial integration, and $W_{n}(R)$ is the probability distribution function specifying the total vector displacements resulting from the $n$ random vector displacements $l_{i}$.

To our knowledge, analytical solutions of this integral have been derived for only a small number of special

* This work was supported in part by a grant from The University of Michigan, Memorial Phoenix Project, and by the U. S. Atomic Energy Commission, Division of Research.

${ }^{1} \mathrm{~J}$. W. Cobble and G. E. Boyd, J. Am. Chem. Soc. 74, 1282 (1952).

2 A. A. Gordus and J. E. Willard, J. Am. Chem. Soc. 79, 4609 (1957).

${ }^{3}$ Lord Rayleigh, Phil. Mag. 37, 321 (1919) or Scientific Papers of Lord Rayleigh (Cambridge University Press, New York, 1920), VI, p. 604.

${ }^{4}$ S. Chandrasekhar, Revs. Modern Phys. 15, 1 (1943). cases. Chandrasekhar ${ }^{4}$ solved the integral for the case of an infinite number of equal-length random displacements. The integrals involving three, four, and six equal-length steps were solved by Rayleigh., Campbell ${ }^{5}$ presented solutions, based on work by O'Connor, ${ }^{6}$ for the integrals involving two and three unequal-length, as well as five equal-length, random vector displacements. ${ }^{7}$ Rayleigh $^{3}$ suggested a method for evaluating $W_{n}(R)$ which is workable in principle, yet in practice becomes so tedious that it might be considered impractical for $n$ greater than about 4 . The direct numerical integration of $\mathrm{Eq}$. (1) was found to be too time-consuming even using an IBM-704 computer. In an attempt to simplify the calculations, we derived a closed general solution for $W_{n}(R)$.

\section{CLOSED GENERAL SOLUTION}

We examine the integral first where $n$ is even, and then where $n$ is an odd number.

\section{Case I: $\boldsymbol{n}$ an Even Number}

Through the use of simple trigonometric relationships, the numerator of the integrand of $\mathrm{Eq}$. (1) can be expressed as a sum of sin terms,

$$
\begin{aligned}
& {\left[\prod_{i=1}^{n} \sin \left(l_{i} \rho\right)\right] \sin (R \rho)} \\
& \qquad=\left(1 / 2^{n}\right) \sum_{j=1}^{2^{n-1}}\left[\sin \left(a_{j} \rho\right)-\sin \left(b_{j} \rho\right)\right],
\end{aligned}
$$

${ }^{5}$ I. G. Campbell, Nucleonika 2, 605 (1957); Chem. Abstr. 52, $11600 \mathrm{i}(1958)$; U. S. Atomic Energy Comm. Nuclear Sci. Abstr. 12, 6505 (1958).

${ }^{6}$ D. O'Connor, Rept. No. $12 / \mathrm{I}-\mathrm{B}$, Polish Acad. Sci., Inst. Nuclear Research, Warsaw; U. S. Atomic Energy Comm. Nuclear Sci. Abstr. 12, 9942 (1958).

${ }^{7}$ The equation presented in the work cited in footnote 5 (apparently obtained from the work cited in footnote 6 ) for three unequal-vector lengths is incomplete. It is applicable only in the case of $\left(l_{1}+l_{2}\right)>l_{3}$. The solution of the integral for the case of $l_{3}>\left(l_{1}+l_{2}\right)$ is somewhat different. At the time of writing we had not received a copy of footnote 6 . 
where both $a_{j}$ and $b_{j}$ are algebraic sums consisting of $(n+1)$ terms of $R, l_{1}, l_{2}, \cdots, l_{n}$. The first quantity in each algebraic sum is always positive and the signs of subsequent l's are determined by the following rules:

1. The number of negative signs in each $a_{j}$ term is even when $n / 2$ is even and odd when $n / 2$ is odd.

2. The number of negative signs in each $b_{j}$ term is even when $n / 2$ is odd and odd when $n / 2$ is even.

Thus, from Eqs. (1) and (2),

$$
W_{n}(R)=\left(2^{n+1} \pi^{2} R \prod_{i=1}^{n} l_{i}\right)^{-1} \sum_{j=1}^{2^{n-1}}\left[\int_{0}^{\infty} \frac{\sin \left(a_{j} \rho\right)}{\rho^{n-1}} d \rho-\int_{0}^{\infty} \frac{\sin \left(b_{j} \rho\right)}{\rho^{n-1}} d \rho\right]
$$

The general integration form of Eq. (3),

$$
\int_{0}^{\infty} \frac{\sin (x \rho)}{\rho^{n-1}} d \rho
$$

can be evaluated by means of integration by parts and is divergent. Since $W_{n}(R)$ is convergent, it is anticipated that the divergency in the integral will disappear upon summing the $a_{j}$ and $b_{j}$ integrals,

$$
\begin{aligned}
& \int_{0}^{\infty} \frac{\sin (x \rho)}{\rho^{n-1}} d \rho=\lim _{y \rightarrow 0, z \rightarrow \infty}\left[x^{n-2} \int_{y}^{z} \frac{\sin (x \rho)}{(x \rho)^{n-1}} x d \rho\right] \\
& =\lim _{y \rightarrow 0, z \rightarrow \infty} x^{n-2}\left\{-\left[\frac{\sin (x \rho)}{(n-2)(x \rho)^{n-2}}+\frac{\cos (x \rho)}{(n-2)(n-3)(x \rho)^{n-3}}\right]\right. \\
& +\left[\frac{\sin (x \rho)}{(n-2)(n-3)(n-4)(x \rho)^{n-4}}+\frac{\cos (x \rho)}{(n-2)(n-3)(n-4)(n-5)(x \rho)^{n-5}}\right] \\
& +\cdots+(-1)^{k}\left[\frac{\sin (x \rho)}{(n-2)(n-3) \cdots(n-2 k)(x \rho)^{n-2 k}}+\frac{\cos (x \rho)}{(n-2)(n-3) \cdots(n-2 k-1)(x \rho)^{n-2 k-1}}\right]+\cdots \\
& \left.+(-1)^{(n / 2)-1}\left[\frac{\sin (x \rho)}{(n-2)(n-3) \cdots(2)(x \rho)^{2}}+\frac{\cos (x \rho)}{(n-2)(n-3) \cdots(2)(1)(x \rho)}\right]\right\}_{\rho=z}^{\rho=y} \\
& +\lim _{y \rightarrow 0, z \rightarrow \infty} \frac{(-1)^{(n / 2)-1}}{(n-2) !}\left[x^{n-2} \int_{z}^{y} \frac{\sin (x \rho)}{x \rho} x d \rho\right] \\
& =\lim _{y \rightarrow 0, z \rightarrow \infty} x^{n-2} \sum_{k=1}^{(n / 2)-1}(-1)^{k}\left[\frac{(n-2 k-1) ! \sin (x \rho)}{(n-2) !(x \rho)^{n-2 k}}+\frac{(n-2 k-2) ! \cos (x \rho)}{(n-2) !(x \rho)^{n-2 k-1}}\right]_{\rho=z}^{\rho=y} \\
& +\frac{(-1)^{(n / 2)-1}}{(n-2) !}\left[\frac{x^{n-2}|x|}{x} \int_{0}^{\infty} \frac{\sin \rho}{\rho} d \rho\right] .
\end{aligned}
$$

Therefore,

$$
\int_{0}^{\infty} \frac{\sin (x \rho)}{\rho^{n-1}} d \rho=\lim _{y \rightarrow 0, z \rightarrow \infty} x^{n-2} \sum_{k=1}^{(n / 2)-1}(-1)^{k}\left[\frac{(n-2 k-1) ! \sin (x \rho)}{(n-2) !(x \rho)^{n-2 k}}+\frac{(n-2 k-2) ! \cos (x \rho)}{(n-2) !(x \rho)^{n-2 k-1}}\right]_{\rho=z}^{\rho=y}+\frac{(-1)^{(n / 2)-1} \pi x^{n-3}|x|}{2(n-2) !} .
$$

On substituting Eq. (4) into Eq. (3),

$$
\begin{aligned}
& W_{n}(R)=\left(2^{n+1} \pi^{2} R \prod_{i=1}^{n} l_{i}\right)^{-1} \sum_{j=1}^{2^{n-1}}\left\{a_{j}^{n-2} \lim _{y \rightarrow 0, z \rightarrow \infty} \sum_{k=1}^{(n / 2)-1}(-1)^{k}\left[\frac{(n-2 k-1) ! \sin \left(a_{j} \rho\right)}{(n-2) !\left(a_{j} \rho\right)^{n-2 k}}+\frac{(n-2 k-2) ! \cos \left(a_{j} \rho\right)}{(n-2) !\left(a_{j} \rho\right)^{n-2 k-1}}\right]\right. \\
& \left.-b_{j}^{n-2} \lim _{y \rightarrow 0, z \rightarrow \infty} \sum_{k=1}^{(n / 2)-1}(-1)^{k}\left[\frac{(n-2 k-1) ! \sin \left(b_{j} \rho\right)}{(n-2) !\left(b_{j} \rho\right)^{n-2 k}}+\frac{(n-2 k-2) ! \cos \left(b_{j} \rho\right)}{(n-2) !\left(b_{j} \rho\right)^{n-2 k-1}}\right]\right\}_{\rho=z}^{\rho=y} \\
& +\left[(-1)^{(n / 2)-1} / 2^{n+2} \pi R(n-2) ! \prod_{i=1}^{n} l_{i}\right]^{2^{n-1}}\left[a_{j=1}^{n-3}\left|a_{j}\right|-b_{j}^{n-3}\left|b_{j}\right|\right] .
\end{aligned}
$$


We now proceed to evaluate the limits of the first two terms on the right-hand side of Eq. (5). These two terms vanish as $\rho$ approaches infinity, thus leaving only the negative limit of these two terms as $\rho$ approaches zero. On calling this limit $S$, we have

$$
\begin{aligned}
S=\sum_{j=1}^{2^{n-1}}\left\{-a_{j}{ }^{n-2}\right. & \lim _{y \rightarrow 0} \sum_{k=1}^{(n / 2)-1}(-1)^{k}\left[\frac{(n-2 k-1) ! \sin \left(a_{j} \rho\right)}{(n-2) !\left(a_{j} \rho\right)^{n-2 k}}+\frac{(n-2 k-2) ! \cos \left(a_{j} \rho\right)}{(n-2) !\left(a_{j} \rho\right)^{n-2 k-1}}\right] \\
& +b_{j}{ }^{n-2} \lim _{y \rightarrow 0} \sum_{k=1}^{(n / 2)-1}(-1)^{k}\left[\frac{(n-2 k-1) ! \sin \left(b_{j} \rho\right)}{(n-2) !\left(b_{j} \rho\right)^{n-2 k}}+\frac{(n-2 k-2) ! \cos \left(b_{j} \rho\right)}{(n-2) !\left(b_{j} \rho\right)^{n-2 k-1}}\right]_{\rho=y}
\end{aligned}
$$

Since the summations are over finite terms, the order of summation may be interchanged. Thus,

$$
\begin{aligned}
S=\lim _{y \rightarrow 0} \sum_{k=1}^{(n / 2)-1}(-1)^{k+1} \frac{(n-2 k-2) !}{(n-2) !}\left[( n - 2 k - 1 ) \sum _ { j = 1 } ^ { 2 ^ { n - 1 } } \left(a_{j}^{2 k-2} \frac{\sin \left(a_{j} \rho\right)}{\rho^{n-2 k}}-\right.\right. & \left.b_{j}{ }^{2 k-2} \frac{\sin \left(b_{j} \rho\right)}{\rho^{n-2 k}}\right) \\
& \left.+\sum_{j=1}^{2^{n-1}}\left(a_{j}^{2 k-1} \frac{\cos \left(a_{j} \rho\right)}{\rho^{n-2 k-1}}-b_{j}{ }^{2 k-1} \frac{\cos \left(b_{j} \rho\right)}{\rho^{n-2 k-1}}\right)\right]_{\rho=y} .
\end{aligned}
$$

However, the terms of the form $\left[\sin \left(W_{j} \rho\right) / \rho^{n-2 k}\right]_{\rho=0}$ are indeterminants. Evaluating them through the use of the l'Hôpital rule, we obtain

$$
\left[\sin \left(W_{j} \rho\right) / \rho^{n-2 k}\right]_{\rho=0}=\left[W_{j} /(n-2 k)\right]\left[\cos \left(W_{j} \rho\right) / \rho^{n-2 k-1}\right]_{\rho=0}
$$

On substituting the form of Eq. (7) into (6), we find

$$
S=\lim _{y \rightarrow 0} \sum_{k=1}^{(n / 2)-1} \frac{(-1)^{k+1}(n-2 k-2) !(2 n-4 k-1)}{(n-2) !(n-2 k)\left(\rho^{n-2 k-1}\right)_{\rho=y}} \sum_{j=1}^{2^{n-1}}\left[a_{j}^{2 k-1} \cos \left(a_{j} \rho\right)-b_{j}{ }^{2 k-1} \cos \left(b_{j} \rho\right)\right]_{\rho=\psi} .
$$

Thus,

$$
S=\sum_{k=1}^{(n / 2)-1} \frac{(-1)^{k+1}(n-2 k-2) !(2 n-4 k-1)}{(n-2) !(n-2 k)\left(\rho^{n-2 k-1}\right)_{\rho=y}} \sum_{j=1}^{2^{n-1}}\left(a_{j}^{2 k-1}-b_{j}^{2 k-1}\right) .
$$

However, if one examines the $a_{j}$ and $b_{j}$ terms, one finds that

$$
\begin{gathered}
\sum_{j=1}^{2^{n-1}}\left(a_{j}-b_{j}\right)=0 \\
\sum_{j=1}^{2^{n-1}}\left(a_{j}{ }^{3}-b_{j}{ }^{3}\right)=0 \\
\cdot \cdot \\
\sum_{j=1}^{2^{n-1}}\left(a_{j}{ }^{n-3}-b_{j}{ }^{n-3}\right)=0 .
\end{gathered}
$$

Therefore,

$$
\sum_{j=1}^{2^{n-1}}\left(a_{j}^{2 k-1}-b_{j}^{2 k-1}\right)=0 \quad \text { for } \quad k=1 \text { to }[(n / 2)-1] .
$$

The successive use of l'Hôpital's rule permits evaluation of the indeterminant. Accordingly,

$$
\begin{array}{r}
\lim _{\rho \rightarrow 0} \frac{1}{\rho^{n-2 k-1}} \sum_{j=1}^{2^{n-1}}\left[a_{j}{ }^{2 k-1} \cos \left(a_{j} \rho\right)-b_{j}{ }^{2 k-1} \cos \left(b_{j} \rho\right)\right]=\lim _{\rho \rightarrow 0} \frac{-1}{(n-2 k-1) \rho^{n-2 k-2}} \sum_{j=1}^{2^{n-1}}\left[a_{j}{ }^{2 k} \sin \left(a_{j} \rho\right)-b_{j}{ }^{2 k} \sin \left(b_{j} \rho\right)\right] \\
=\lim _{\rho \rightarrow 0} \frac{-1}{(n-2 k-1)(n-2 k-2) \rho^{n-2 k-3}} \sum_{j=1}^{2^{n-1}}\left[a_{j}^{2 k+1} \cos \left(a_{j} \rho\right)-b_{j}{ }^{2 k+1} \cos \left(b_{j} \rho\right)\right]
\end{array}
$$


$=\cdots$ etc. Consequently, for any specific value of $k$, the process is continued until the denominator becomes $\rho$ times a constant. Under these conditions $\rho=\rho^{n-2 k-2 m+1}$ and the indeterminant becomes

$\lim _{\rho \rightarrow 0} \frac{(-1)^{m} \sum_{j=1}^{2^{n-1}}\left[a_{j}^{2 k+2 m-1} \cos \left(a_{j} \rho\right)-b_{j}{ }^{2 k+2 m-1} \cos \left(b_{j} \rho\right)\right]}{(n-2 k-1)(n-2 k-2) \cdots(n-2 k-2 m+2) \rho^{n-2 k-2 m+1}}=\frac{(-1)^{m}(n-2 k-2 m+1) !}{\rho(n-2 k-1) !} \sum_{j=1}^{2^{n-1}}\left(a_{j}^{2 k+2 m-1}-b_{j}^{2 k+2 m-1}\right)$.

Since $\rho^{n-2 k-2 m+1}=\rho$, then

when

$$
n-2 k-2 m+1=1 \text { and } m=\frac{1}{2}(n-2 k),
$$

Thus,

$$
k=1, \quad m=\frac{1}{2}(n-2) \quad \text { and } \quad 2 k+2 m-1=n-1 .
$$

$$
\sum_{j=1}^{2^{n-1}}\left(a_{j}^{2 k+2 m-1}-b_{j}^{2 k+2 m-1}\right)=0
$$

For any value of $k$,

$$
2 k+2 m-1=2 k+n-2 k-1=n-1 .
$$

Therefore, this summation is always equal to zero. Thus, Eq. (9) remains an indeterminant. However, further use of l'Hôpital's rule leads to

$\lim _{\rho \rightarrow 0} \frac{1}{\rho^{n-2 k-1}} \sum_{j=1}^{2^{n-1}}\left[a_{j}^{2 k-1} \cos \left(a_{j} \rho\right)-b_{j}^{2 k-1} \cos \left(b_{j} \rho\right)\right]=\left[\frac{(-1)^{[(n-2) / 2]+1}}{(n-2 k-1) !} \sum_{j=1}^{2^{n-1}}\left[a_{j}^{n} \sin \left(a_{j} \rho\right)+b_{j}^{n} \sin \left(b_{j} \rho\right)\right]\right]_{\rho=0}=0$.

Therefore, Eq. (8) yields

$$
S=0
$$

or

$$
W_{n}(R)=\frac{(-1)^{(n / 2)-1}}{2^{n+2} \pi R(n-2) ! \prod_{i=1}^{n} l_{i}} \sum_{j=1}^{2^{n-1}}\left(a_{j}^{n-3}\left|a_{j}\right|-b_{j}^{n-3}\left|b_{j}\right|\right)
$$

When $n / 2$ is even, $(-1)^{(n / 2)-1}=-1$; when $n / 2$ is odd, this term $=+1$.

However, according to the rules defining the $a_{j}$ and $b_{j}$ terms, it is found that $a_{j}$ terms for $n / 2$ equal to an even number are the same as $b_{j}$ terms for $n / 2$ equal to an odd number, and vice versa. Therefore, Eq. (10) may be stated in a general manner irrespective of the evenness or oddness of $n / 2$.

Hence, the general solution for $n$ equal to an even number is

$$
W_{n}(R)=\left[2^{n+2} \pi R(n-2) ! \prod_{i=1}^{n} l_{i}\right]^{-1} \sum_{j=1}^{2^{n-1}}\left(a_{j}^{n-3}\left|a_{j}\right|-b_{j}^{n-3}\left|b_{j}\right|\right)
$$

where

1. both $a_{j}$ and $b_{j}$ are algebraic sums consisting of $(n+1)$ terms of $R, l_{1}, l_{2}, \cdots, l_{n}$;

2. there are a total of $2^{n-1}$ different $a_{j}$ and $2^{n-1}$ different $b_{j}$ terms;

3 . the first quantity in each $a_{j}$ or $b_{j}$ term is always positive;

4. the total number of negative signs in each $a_{j}$ term is an odd number, $[1,3,5, \cdots,(n-1)]$;

5 . the total number of negative signs in each $b_{j}$ term is an even number, $[0,2,4, \cdots, n]$.

\section{Case II: $\boldsymbol{n}$ an Odd Number}

The deriviation is similar to that of Case I. By using simple trigonometric relationships, the numerator of the integrand of Eq. (1) is expressed as a series of cosine terms,

$$
\left[\prod_{i=1}^{n} \sin \left(l_{i} \rho\right)\right] \sin (R \rho)=\left(1 / 2^{n}\right) \sum_{j=1}^{2^{n-1}}\left[\cos \left(c_{j} \rho\right)-\cos \left(d_{j} \rho\right)\right]
$$


where both $c_{j}$ or $d_{j}$ consist of $(n+1)$ terms of $R, l_{1}, l_{2}, \cdots, l_{n}$. The first quantity $R$ in each term is always positive and the signs of subsequent $l$ 's are determined by the following rules:

(1) The number of negative signs in each $c_{j}$ term is even when $(n-1) / 2$ is even and odd when $(n-1) / 2$ is odd.

(2) The number of negative signs in each $d_{j}$ term is even when $(n-1) / 2$ is odd and odd when $(n-1) / 2$ is even. Thus, from Eqs. (1) and (12),

$$
W_{n}(R)=\left(2^{n+1} \pi^{2} R \prod_{i=1}^{n} l_{i}\right)^{-1} \sum_{j=1}^{2^{n-1}}\left(\int_{0}^{\infty} \frac{\cos \left(c_{j} \rho\right)}{\rho^{n-1}} d \rho-\int_{0}^{\infty} \frac{\cos \left(d_{j} \rho\right)}{\rho^{n-1}} d \rho\right) .
$$

The general integration form of Eq. (13) is

$$
\int_{0}^{\infty} \frac{\cos (x \rho)}{\rho^{n-1}} d \rho
$$

which is divergent.

As in Case I, integration by parts results in

$$
\begin{aligned}
\int_{0}^{\infty} \frac{\cos (x \rho)}{\rho^{n-1}} d \rho=x^{n-2} \lim _{y \rightarrow 0, z \rightarrow \infty}\left\{\sum_{k=1}^{(n-1) / 2}\left[\frac{(-1)^{k}(n-2 k) ! \sin (x \rho)}{(n-2) !(x \rho)^{n-2 k+1}}+\frac{(-1)^{k}(n-2 k-1) ! \cos (x \rho)}{(n-2) !(x \rho)^{n-2 k}}\right]+\frac{\sin (x \rho)}{(x \rho)^{n-1}}\right\} \\
+\left[(-1)^{(n-1) / 2} \pi / 2(n-2) !\right] x^{n-3}|x|
\end{aligned}
$$

This general solution (14) may be utilized in Eq. (13). The indeterminate" expression which results is evaluated as in Case I, using the rule of l'Hôpital successively. 'The final result is, for $(n-1) / 2$ equal to an even number,

$$
W_{n}(R)=\left[2^{n+2} \pi R(n-2) ! \prod_{i=1}^{n} l_{i}\right]^{-1} \sum_{j=1}^{2^{n-1}}\left(c_{j}^{n-3}\left|c_{j}\right|-d_{j}^{n-3}\left|d_{j}\right|\right) .
$$

For $(n-1) / 2$ equal to an odd number, $W_{n}(R)$ is equal to the negative of Eq. (15). Reciprocity relationships again exist so that $c_{j}$ 's for $(n-1) / 2$ equal to an even number are the same as $d_{j}^{\prime}$ s for $(n-1) / 2$ equal to an odd number. Thus, the over-all expression is identical for both $(n-1) / 2$ even or odd, and for $n$ equal to an odd number the solution is Eq. (15). The restrictions are:

1 . both $c_{j}$ and $d_{j}$ are algebraic sums consisting of $(n+1)$ terms of $R, l_{1}, l_{2}, \cdots, l_{n}$;

2. there are a total of $2^{n-1}$ different $c_{j}$ and $2^{n-1}$ different $d_{j}$ terms;

3. the first quantity in each $c_{j}$ or $d_{j}$ term is always positive;

4. the total number of negative signs in each $c_{j}$ term is an odd number, $[1,3,5, \cdots, n]$;

5. the total number of negative signs in each $d_{j}$ term is an even number, $[0,2,4, \cdots,(n-1)]$.

\section{General Solution}

On comparing Eqs. (11) and (15) and their respective conditions, it is seen that the solution is of the same general form, with conditions 4 and 5 differing only slightly. It is therefore possible to combine these two cases in the form of one general equation valid for any finite value of $n \geq 2$. Thus,

$$
\begin{aligned}
& W_{n}(R) \\
& =\left[2^{n+2} \pi R(n-2) ! \prod_{i=1}^{n} l_{i}\right]^{-1} \sum_{j=1}^{2^{n-1}}\left(M_{j}^{n-3}\left|M_{j}\right|-N_{j}^{n-3}\left|N_{j}\right|\right),
\end{aligned}
$$

where

1. both $M_{j}$ and $N_{j}$ are algebraic sums consisting of $(n+1)$ terms of $R, l_{1}, l_{2}, \cdots, l_{n}$;

2. ther are a total of $2^{n-1}$ different $M_{j}$ and $2^{n-1}$ different $N_{j}$ terms;

3. the first quantity in each $M_{j}$ or $N_{j}$ term is always positive;

4. the total number of negative signs in each $M_{j}$ term is an odd number, $\left[1,3,5, \cdots,(n-1)_{e}\right.$ or $\left.(n)_{0}\right]$;

5 . the total number of negative signs in each $N_{j}$ term is an even number, $\left[0,2,4, \cdots,(n)_{e}\right.$ or $\left.(n-1)_{0}\right]$, where $e$ and $o$ represent $n$ equal to an even or odd number, respectively.

Given in the Appendix are the complete solutions for $n=2,3$, and 4 unequal-length random vector displacements.

\section{Equal-Length Displacements}

A case of particular interest occurs when all displacements are of equal length. The probability distribution function, Eq. (1), is therefore

$$
W_{n}(R)=\left(2 \pi^{2} R l^{n}\right)^{-1} \int_{0}^{\infty}\left\{\left[\sin ^{n}(\rho l)\right] \sin (R \rho) / \rho^{n-1}\right\} d \rho .
$$

We examine the general solution, Eq. (16). If we choose $R$ as the first quantity in the $M_{j}$ and $N_{j}$ terms, the remaining quantities are all $l$ 's. According to the condition for $M_{j}$, the number of negative $l$ 's is odd, $\left[1,3,5, \cdots,(2 s-1), \cdots,(n-1)_{e}\right.$ or $\left.(n)_{0}\right]$. Therefore, 
the number of positive $l$ 's in any $M_{j}$ term is $n-(2 s-1)$, where

$$
s=1,2,3, \cdots,(n / 2)_{e} \text { or }[(n+1) / 2]_{o} .
$$

As a result, the net number of $l$ 's is

$$
n-(2 s-1)-(2 s-1)=n+2-4 s,
$$

and $M_{s}=R+(n+2-4 s) l$.
The number of possible combinations of positive $l$ 's and negative $l$ 's that will result in $M_{s}$ [i.e., $n$ total $l$ 's, of which $(2 s-1)$ are negative] is the binomial coefficient

$$
\left(\begin{array}{c}
n \\
2 s-1
\end{array}\right)
$$

Therefore,

$$
\sum_{j=1}^{2^{n-1}} M_{j}^{n-3}\left|M_{j}\right|=\sum_{s=1}^{(n / 2)_{e} \text { or }[(n+1) / 2]_{o}}\left(\begin{array}{c}
n \\
2 s-1
\end{array}\right) M_{s}^{n-3}\left|M_{s}\right| .
$$

Regarding the $N_{j}$ terms, the number of negative $l^{\prime}$ s is even, $\left[0,2,4, \cdots,(2 s-2), \cdots,(n)_{e}\right.$ or $\left.(n-1)_{o}\right]$ where $s=1,2,3, \cdots,[(n / 2)+1]_{e}$ or $[(n+1) / 2]_{0}$. Therefore, in a manner similar to that above, the net number of $l$ 's in any $N_{s}$ term is: $n-(2 s-2)-(2 s-2)=n+4-4 s$ and $N_{s}=R+(n+4-4 s) l$. The number of possible combinations of positive l's and negative $l$ 's that will result in $N_{s}$ is

$$
\left(\begin{array}{c}
n \\
2 s-2
\end{array}\right)
$$

Therefore,

$$
\sum_{j=1}^{2^{n-1}} N_{j}^{n-3}\left|N_{j}\right|=\sum_{s=1}^{[(n / 2)+1]} \sum_{\text {or }[(n+1) / 2]}\left(\begin{array}{c}
n \\
2 s-2
\end{array}\right) N_{s}^{n-3}\left|N_{s}\right| .
$$

The probability distribution function is

$$
\begin{aligned}
& W_{n}(R)=\left[2^{n+2} \pi(n-2) ! R l^{n}\right]^{-1}\left\{\sum_{s=1}^{(n / 2)_{e} \text { or }[(n+1) / 2]_{o}}\left(\begin{array}{c}
n \\
2 s-1
\end{array}\right)[R+(n+2-4 s) l]^{n-3}|R+(n+2-4 s) l|\right. \\
& \left.-\sum_{s=1}^{[(n / 2)+1]_{o}^{\text {or }}[(n+1) / 2]_{0}}\left(\begin{array}{c}
n \\
2 s-2
\end{array}\right)[R+(n+4-4 s) l]^{n-3}|R+(n+4-4 s) l|\right\} \text {. }
\end{aligned}
$$

Equations (16) and (20), when reduced to special cases, result in expressions identical to those stated in the literature. ${ }^{4,5}$

\section{Evaluation of Probability}

The probability that the total displacement is between 0 and $R$ as a result of $n$ random vector displacements is,

$$
P_{n}(R)=\int_{0}^{R} 4 \pi R^{2} W_{n}(R) d R
$$

The probability distribution is,

$$
d P_{n}(R) / d R=4 \pi R^{2} W_{n}(R) .
$$

\section{COMPARISON WITH APPROXIMATIONS}

\section{Equal Random Steps}

A Gaussian distribution is perhaps the most commonly utilized approximation of the probability distribution $(P D)$, Eq. (22). In this case, ${ }^{4}$

$$
W_{n}(R)=\left[1 /\left(2 \pi n\left\langle l^{2}\right\rangle_{\mathrm{AV}} / 3\right)^{\frac{3}{2}}\right] \exp \left[-3 R^{2} / 2 n\left\langle l^{2}\right\rangle_{\mathrm{AV}}\right] \text {. }
$$

Figure 1 is the percent error in the Gaussian ap- proximation of the probability distribution for $n=3,5$, and 10 equal-length random vector displacements. For this plot: percent error $=100\left[(P D)_{\mathrm{ex}}-(P D)_{g}\right] /(P D)_{\mathrm{ex}}$, where $(P D)_{\text {ex }}$ is the exact and $(P D)_{\circ}$ the Gaussian calculated value.

Similarly, Fig. 2 is the percent error in the Gaussian approximation of the probability for $n=3,5$, and 10 equal-length random vector displacements. Here: percent error $=100\left[P_{\text {ex }}-P_{g}\right] / P_{\text {ex }}$, where the subscripts have the same meaning as above.

\section{Five Unequal Steps}

To illustrate the error in various approximations of an unequal-step random walk we have chosen five steps of fractional lengths: $0.582,0.135,0.131,0.092$, and 0.060 . These five steps are characteristic of the relative magnitudes of the gamma rays emitted following neutron capture. Specifically, these steps correspond to the $5.01,1.16,1.13,0.79$, and $0.51 \mathrm{Mev}$ gamma-ray cascade in the $\mathrm{Cl}^{35}(n, \gamma) \mathrm{Cl}^{36}$ process discussed below.

Figure 3 illustrates the probability distribution calculated via (a) the exact expression, Eqs. (16) and (22); (b) Eq. (20), assuming each of the five steps to be of equal length, $0.2 R_{\max }$; (c) the Gaussian distribution for $\left\langle l_{i}{ }^{2}\right\rangle_{A v}=0.0771$; and (d) a modified Gaussian dis- 


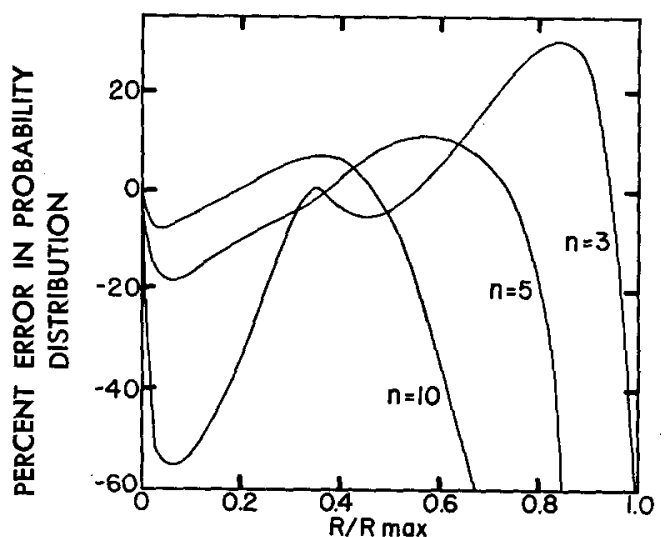

FIG. 1. Percent error in probability distribution for a Gaussian distribution vs the fraction of the maximum possible resultant vector length for three, five, and 10 equal-length random vectors.

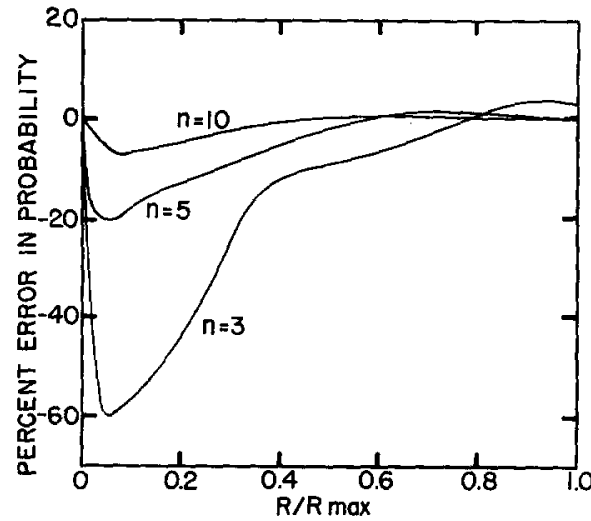

FIG. 2. Percent error in probability for a Gaussian distribution vs the fraction of the maximum possible resultant vector length for three, five, and 10 equal-length random vectors.

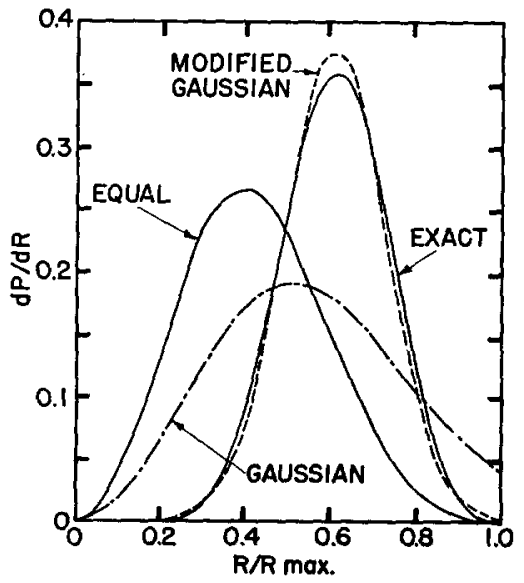

FIG. 3. Probability distribution of gamma-ray energy (in units of $\mathrm{Mev}^{-1}$ ) calculated according to different methods vs the fraction of the maximum possible resultant gamma-ray energy for five random gamma rays. Refer to text for individual gamma-ray energies.

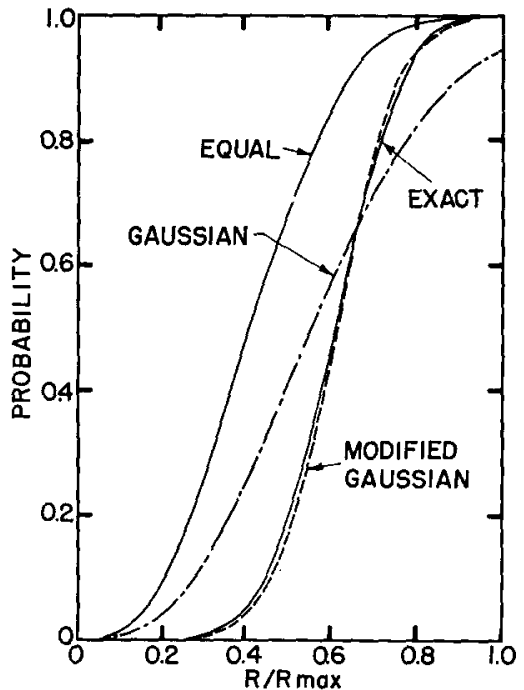

Fig. 4. Probability of gamma-ray energy calculated according to different methods vs the fraction of the maximum possible resultant gamma-ray energy for five random gamma rays. Refer to text for individual gamma-ray energies.

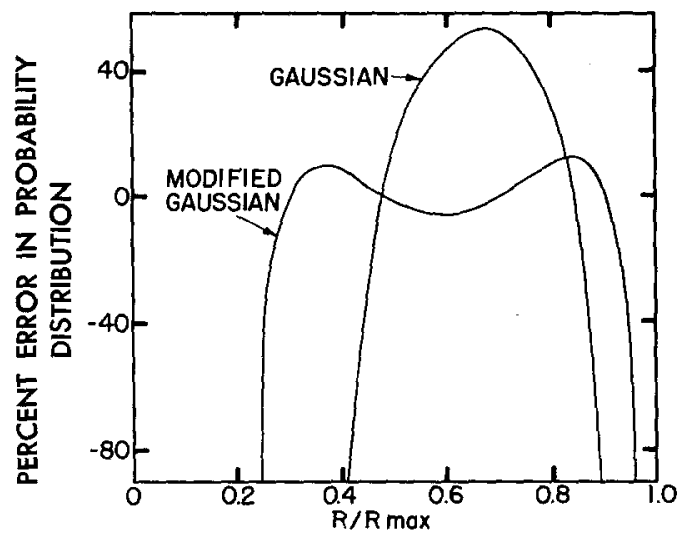

FIG. 5. Percent error in probability distribution in terms of the exact calculation vs the fraction of the maximum possible resultant gamma-ray energy for the approximate solutions given in Fig. 3.

TABLE I. Gamma-ray cascades ${ }^{\mathbf{8}}$ from $\mathrm{Cl}^{35}(n, \gamma) \mathrm{Cl}^{36}$

\begin{tabular}{lc}
\hline & \\
Energies (Mev) & \% Occurrence \\
\hline 8.55 & 3.71 \\
$7.78,0.79$ & 10.33 \\
$7.40,1.16$ & 18.54 \\
$6.96,1.60$ & 2.52 \\
$6.64,1.95$ & 15.23 \\
$5.72,2.87$ & 3.84 \\
$5.28,3.34$ & 17.10 \\
$6.64,1.16,0.79$ & 11.26 \\
$6.15,0.51,1.95$ & 7.42 \\
$5.01,1.65,1.95$ & 2.12 \\
$6.15,0.51,1.16,0.79$ & 2.38 \\
$5.01,1.13,0.51,1.95$ & 1.59 \\
$5.01,1.65,1.16,0.79$ & 3.18 \\
$5.01,1.13,0.51,1.16,0.79$ & 0.79 \\
\hline
\end{tabular}

a See footnote 12 .

b Based on Table I of work cited in footnote 12 (b). 


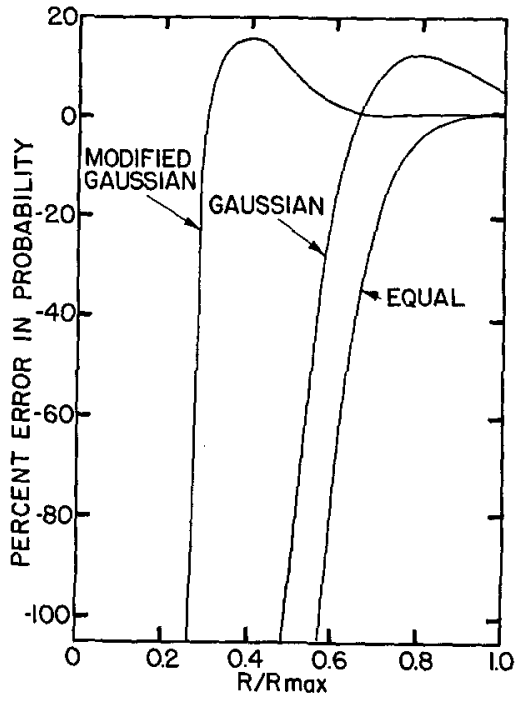

Fig. 6. Percent error in probability in terms of the exact calculation vs the fraction of the maximum possible resultant gamma-ray energy for approximate solutions given in Fig. 4.

tribution in which the four small steps are approximated by a Gaussian distribution with $\left\langle l_{i}{ }^{2}\right\rangle_{\mathrm{AV}}=0.0119$ and the large step, $l_{1}=0.582$, is combined with the Gaussian function. ${ }^{8}$ Figure 4 is the probability for these same four calculations.

The percent errors in the Gaussian and modified Gaussian approximations relative to the exact calculation for the probability distribution (Fig. 3) are depicted in Fig. 5. Similarly, the percent errors in the Gaussian, modified Gaussian, and five equal-step approximations relative to the exact calculation for the probability (Fig. 4) are depicted in Fig. 6.

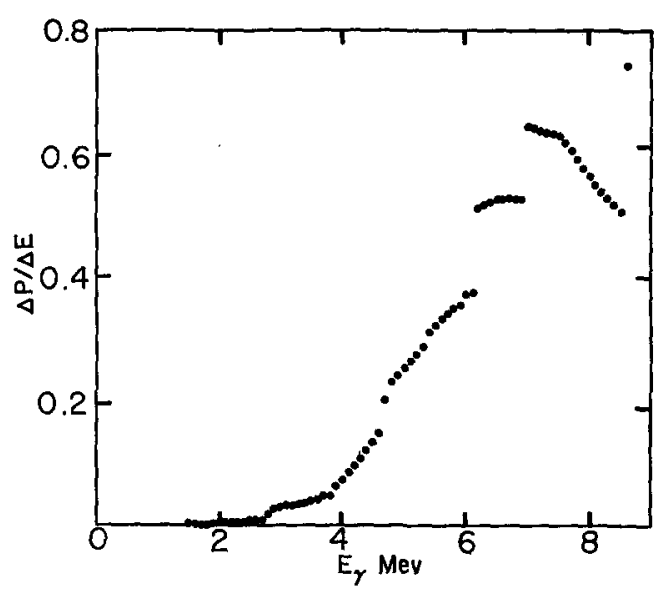

FIG. 7. Summed probability distribution of gamma-ray energies (in units of $\mathrm{Mev}^{-1}$ ) vs the net gamma-ray energy for the 14 gamma cascades of the $\mathrm{Cl}^{35}(n, \gamma) \mathrm{Cl}^{36}$ process as given in Table $\mathrm{I}$.

It is occasionally necessary to utilize the distribution function in various theoretical investigations. For example, we anticipate a need for the gamma-ray recoil energy distribution in evaluating the high energy kinetics of chemical reactions activated by nuclear transformations. ${ }^{2}$ Therefore, it is desirable to determine whether any of the mathematically simplified randomwalk approximations are sufficiently in agreement with the exact calculation to permit their use. As noted in Figs. 5 and 6, for this five-step gamma cascade, the modified Gaussian distribution serves as the best approximation.

The modified Gaussian is calculated as follows: The large step is designated $l_{1}$ and, according to the original form of the Markoff process, ${ }^{4}$

$$
W_{n}(R)=\left(8 \pi^{3}\right)^{-1} \int_{-\infty}^{+\infty} \exp (i \boldsymbol{\varrho} \cdot \mathbf{R}) A_{n}(\rho) d \mathbf{\varrho}
$$

where, for the remaining $(n-1)$ steps,

$$
A_{n-1}(\rho)=\prod_{j=2}^{n} \int_{-\infty}^{+\infty} \tau_{j}\left(r_{j}\right) \exp \left(i_{\mathbf{0}} \cdot \mathbf{r}_{j}\right) d \mathbf{r}_{j} .
$$

For a Gaussian distribution,

Thus,

$$
\tau_{j}\left(\boldsymbol{r}_{j}\right)=\left[1 /\left(2 \pi l_{j}^{2} / 3\right)^{\frac{3}{2}}\right] \exp \left(-3\left|\boldsymbol{r}_{j}\right|^{2} / 2 l_{j}^{2}\right) .
$$

$$
A_{n-1}(\rho)=\exp \left[-\left(\rho^{2} \sum_{j=2}^{n} l_{j}^{2}\right) / 6\right]
$$

and

Therefore,

$$
A_{1}=\sin \left(\rho l_{1}\right) / \rho l_{1}
$$

$$
A_{n}=A_{1} \cdot A_{n-1}
$$

and

$$
\begin{array}{r}
W_{n}(R)=\left(8 \pi^{2} R\right)^{-1} \int_{-\infty}^{+\infty} \frac{\sin (\rho R) \sin \left(\rho l_{1}\right)}{\rho l_{1}} \exp \left[-\rho^{2}(n-1)\left\langle l^{2}\right\rangle_{\mathrm{AV}} / 6\right] \rho d \rho \\
W_{n}(R)=\frac{1}{8 \pi^{\frac{3}{3}} R l_{1}\left[(n-1)\left\langle l^{2}\right\rangle_{\mathrm{AV}} / 6\right]^{\frac{1}{2}}}\left\{\exp \left[\frac{-3\left(R-l_{1}\right)^{2}}{2(n-1)\left\langle l^{2}\right\rangle_{\mathrm{AV}}}\right]-\exp \left[\frac{-3\left(R+l_{5}\right)^{2}}{2(n-1)\left\langle l^{2}\right\rangle_{\mathrm{AV}}}\right]\right\}
\end{array}
$$

\footnotetext{
${ }^{8}$ The authors are indebted to Professor G. E. Uhlenbeck for suggesting this approximation.
} 


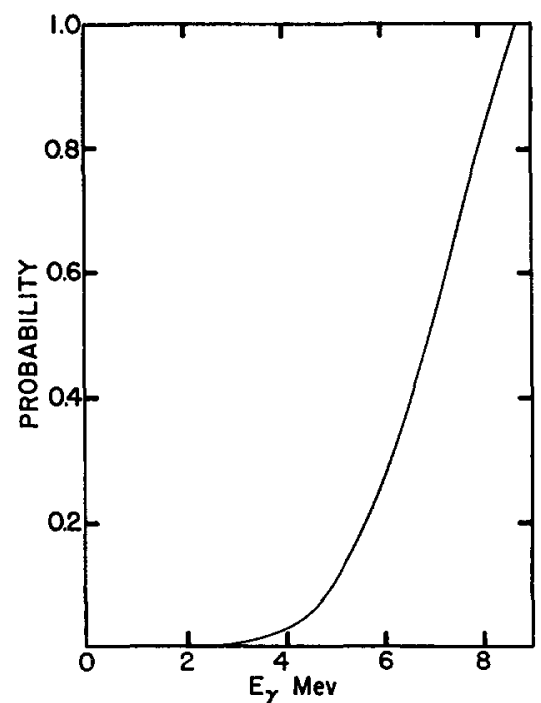

FIG. 8. Summed probability of gamma-ray energies vs the net gamma-ray energy for the 14 gamma cascades of the $\mathrm{Cl}^{35}(n, \gamma) \mathrm{Cl}^{36}$ process as given in Table I.

\section{Cl $^{36}$ GAMMA RECOIL}

Our original purpose in evaluating the random-walk expression was to obtain data regarding the distribution of recoil energy imparted to an atom as a result of cascade-gamma emission in $(n, \gamma)$ activation processes, ${ }^{9,10}$ Such information is needed in order to

TABLE II. Probability distribution function for three unequal-length random steps.

\begin{tabular}{ccc}
\hline$R$-range & Applicability & $W_{3}(R)$ \\
\hline $0 \rightarrow(A-B-C)$ & 1 & 0 \\
$0 \rightarrow(-A+B+C)$ & 2 & $\frac{1}{8 \pi A B C}$ \\
$(A-B-C) \rightarrow(A-B+C)$ & 1 \\
$(-A+B+C) \rightarrow(A-B+C)$ & $2)$ & $\frac{R-A+B+C}{16 \pi R A B C}$ \\
$(A-B+C) \rightarrow(A+B-C)$ & 1 or 2 & $\frac{1}{8 \pi R A \bar{B}}$ \\
$(A+B-C) \rightarrow(A+B+C)$ & 1 or 2 & $\frac{-R+A+B+C}{16 \pi R A B C}$ \\
\hline
\end{tabular}

a 1 is where $A>(B+C)$; 2 is where $A<(B+C)$.

9 This type of calculation recently has been considered by Zvara (footnote 10 ) for the $\mathrm{Cl}^{35}(n, \gamma) \mathrm{Cl}^{36}$ process. Zvara, however, limits the calculations to cascades involving only two and three gamma rays. He approximates a cascade of four gamma rays by a three-step calculation. Zvara performed the calculations in terms of the recoil energy of the activated atom. Therefore, his equations can be transformed into the form given in this paper by an appropriate change in the variable.

10 I. Zvara, Vestnik Moskov. Univ., Ser. Mat., Mekhan. Astron., Fiz. i Khim. 13, No. 6, 127 (1958); Chem. Abstr. 53, $15083 f$ (1959); U. S. Atomic Energy Comm. Nuclear Sci. Abstr. 14, $3046(1960)$.

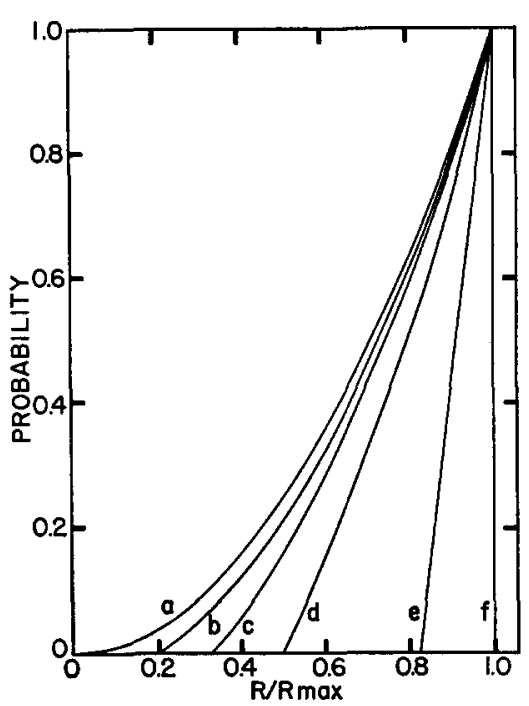

FIG. 9. Probability vs the fraction of the maximum possible resultant vector length for various random walks involving the two steps: $A$ and $B$, (a) $A=B$, (b) $A=\frac{3}{2} B$, (c) $A=2 B$, (d) $A=3 B$, (e) $A=10 B$, (f) $B=0$.

evaluate more quantitatively hot-atom reaction mechanisms. ${ }^{11}$ In addition, this information is of importance in investigations of the failure to bond rupture following neutron activation, ${ }^{2}$ where the fraction of $(n, \gamma)$ activated atoms which fail to dissociate from their parent compound should be related to the fraction of the atoms which receive a gamma-recoil energy less than some threshold energy.

Unfortunately, to date very little neutron-capture

TABLE III. Various cases for four unequal-length steps.

\begin{tabular}{rl}
\hline \hline Case & \multicolumn{1}{c}{ Conditions $^{\mathbf{a}}$} \\
\hline 1 & $A>(B+C+D)$ and $B>(C+D)$ \\
2 & $A>(B+C+D)$ and $(C+D)>B$ \\
3 & $(A+D)>(B+C),(B+D)>A$, and $B>(C+D)$ \\
4 & $(A+D)>(B+C),(B+C)>A, A>(B+D)$, and \\
& $B>(C+D)$ \\
5 & $(B+C+D)>A, A>(B+C)$, and $B>(C+D)$ \\
6 & $(A+D)>(B+C)$ and $(C+D)>A$ \\
7 & $(A+D)>(B+C)$ and $(B+D)>A>(C+D)>B$ \\
8 & $(A+D)>(B+C)>A>(B+D)$ and $(C+D)>B$ \\
9 & $(B+C+D)>A>(B+C)$ and $(C+D)>B$ \\
10 & $(B+C)>(A+D),(B+D)>A$, and $B>(C+D)$ \\
11 & $(B+C)>(A+D), A>(B+D)$, and $B>(C+D)$ \\
12 & $(B+C)>(A+D)$ and $(C+D)>A$ \\
13 & $(B+C)>(A+D)$ and $(B+D)>A>(C+D)>B$ \\
14 & $(B+C)>(A+D), A>(B+D)$, and $(C+D)>B$ \\
\hline \hline
\end{tabular}

a Where $A>B>C>D$.

${ }^{1}$ J. E. Willard, Ann. Rev. Phys. Chem. 6, 141 (1955). 


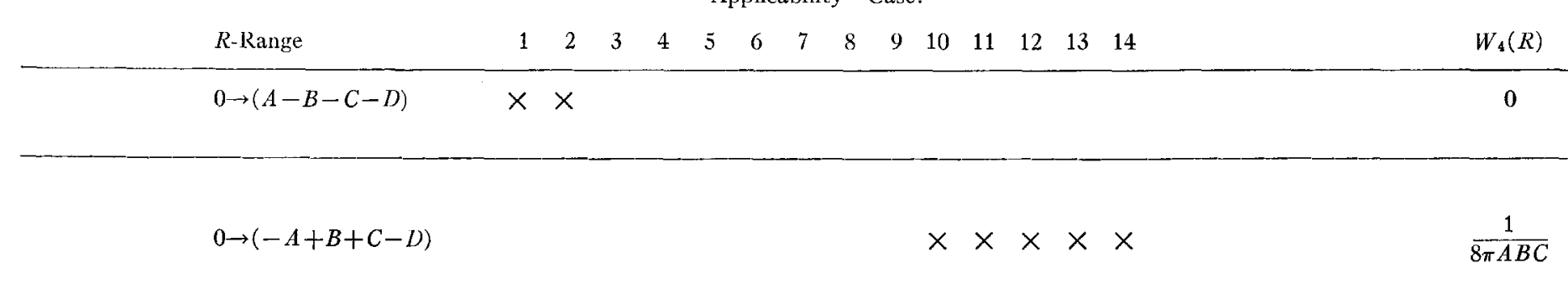

\begin{tabular}{c}
$0 \rightarrow(-A+B+C+D)$ \\
\hline $0 \rightarrow(A-B-C+D)$ \\
\hline$(A-B-C-D) \rightarrow(A-B-C+D)$ \\
\hline$(-A+B+C+D) \rightarrow(A-B-C+D)$
\end{tabular}

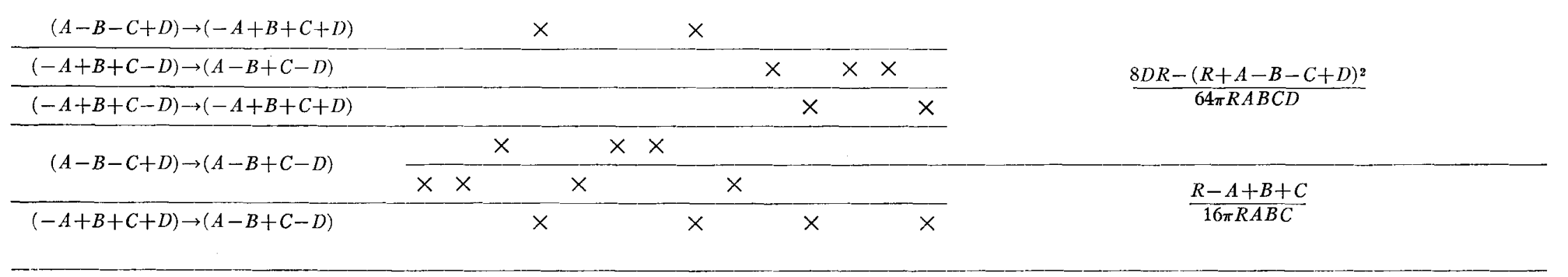

$\frac{(A-B+C-D) \rightarrow(-A+B+C+D)}{(A-B+C-D) \rightarrow(A+B-C-D)} \times \frac{\times}{\times} \times \frac{4 C D-(A-B)^{2}-(R-C-D)^{2}}{32 \pi R A B C D}$




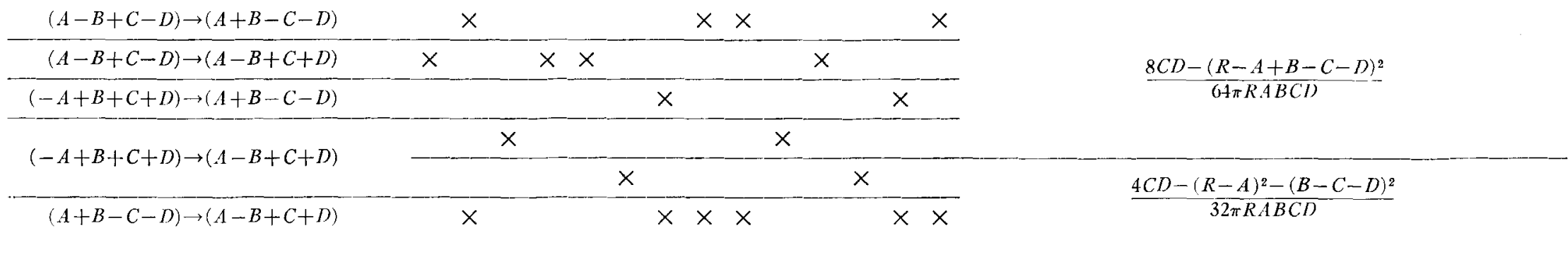
$(A-B+C+D) \rightarrow(A+B-C-D)$
$\times \quad \times \times$
$\times \quad \times$
1
$\overline{8} \pi R A B$

$\frac{(A+B-C-D) \rightarrow(A+B-C+D)}{(A-B+C+D) \rightarrow(A+B-C+D)} \times \frac{\times \times}{\times} \times \frac{\times}{\times \times \times} \times \frac{}{\times \times}$

$8 C D-(R-A-B+C+D)^{2}$ $64 \pi R A B C D$

$(A+B-C+D) \rightarrow(A+B+C-D) \quad \times \times \times \times \times \times \times \times \times \times \frac{A+B+C-R}{16 \pi R A B C}$

\footnotetext{
$(A+B+C-D) \rightarrow(A+B+C+D) \quad \times \times \times \times \times \times \times \times \times \times \times \times \times \times$
}

$$
\frac{(R-A-B-C-D)^{2}}{64 \pi R A B C D}
$$


gamma-ray data are available and most of what has appeared in the literature is incomplete. One process, which has been studied in great detail, however, is the $\mathrm{Cl}^{35}(n, \gamma) \mathrm{Cl}^{36}$ reaction. The neutron-capture gamma-ray emission is spherically symmetrical and the gamma-ray scheme is fairly complex. We have chosen this activation process to illustrate a particular use of the previously derived random-walk (or to be more precise for this application: random flight) equation.

The random-walk equation requires the use of momentum vectors. However, in the particular case of gamma-ray emission the energy of a gamma ray differs from the momentum by only a multiplicative constant (the velocity of light). Therefore, the gammaray energies were used in the calculation.

We utilized the data of Groshev et $a{ }^{12}$, arbitrarily limiting the possible gamma cascades to the 14 listed in Table I. For convenience and accuracy, the 14 gammaray energy probability distributions and probabilities were calculated separately with the aid of an IBM-704 computer and then added according to the abundances given in Table I. It should be emphasized, however, that these calculations could have been performed with little effort using a desk calculator. The summed probability distribution of gamma-ray energies is depicted in Fig. 7 , the summed probability in Fig. 8.

It is of interest to note the "step function" nature of the results of Fig. 7. For example, the jump at 6.24 $\mathrm{Mev}$ is a result of the initial contribution from the $7.40+1.16 \mathrm{Mev}$ gamma cascade. The jump at 6.99 Mev results from the $7.78+0.79 \mathrm{Mev}$ cascade.

\section{APPENDIX}

Presented below are the complete solutions of $W_{n}(R)$ for $n=2,3$, and 4 random-length vectors calculated via Eq. (16). It should be noted that the signs and magnitudes of the $M_{j}$ and the $N_{j}$ terms vary as a result of variations in $R$ and as a result of these variations, $W_{n}(R)$ is a piecewise continuous curve with respect to $R$. Consequently $W_{n}(R)$, and thus $d P_{n}(R) / d R$ and $P_{n}(R)$, must be evaluated in segments.

\section{Two Random Steps}

For two random-length vectors, $l_{1}=A$ and $l_{2}=B$ where $A>B$, the solutions are trivial. There are $2^{2-1}=$

${ }^{12}$ (a) L. V. Groshev, B. P. Adyasevich, and A. M. Demidov, Proc. Intern. Conf. Peaceful Uses Atomic Energy, Geneva, 1955 (United Nations, New York, 1956); (b) R. E. Segel, Phys. Rev. 113, 844 (1959).
$2 M_{j}$ and $2 N_{j}$ terms. They are $M_{1}=R-A+B, M_{2}=$ $R+A-B, N_{1}=R+A+B, N_{2}=R-A-B . W_{2}(R)=0$ in the range $0<R<(A-B)$, and $=1 /(8 \pi R A B)$ in the range $(A-B)<R<(A+B)$.

Depicted in Fig. 9 are the probabilities for various two-step processes.

\section{Three Random Steps}

For the process involving three random steps there exist two possible cases. Assuming $\left(l_{1}=A\right)>\left(l_{2}=B\right)>$ $\left(l_{3}=C\right)$, then these two cases are: 1 . Where $A>(B+$ $C$; or), 2. Where $A<(B+C)$.

The four $M_{j}$ and four $N_{j}$ terms are:

$$
\begin{array}{cc}
M_{j} & N_{j} \\
M_{1}=R+A+B-C & N_{1}=R+A+B+C \\
M_{2}=R+A-B+C & N_{2}=R+A-B-C \\
M_{3}=R-A+B+C & N_{3}=R-A+B-C \\
M_{4}=R-A-B-C & N_{4}=R-A-B+C .
\end{array}
$$

As an illustration, let us consider the calculation of $W_{3}(R)$ in the range $(A+B-C) \rightarrow(A+B+C)$, the solution being valid for both cases (1) and (2).

$$
\begin{aligned}
W_{3}(R) & =(32 \pi R A B C)^{-1} \sum_{j=1}^{j=4}\left(\left|M_{j}\right|-\left|N_{j}\right|\right) \\
& =\frac{M_{1}+M_{2}+M_{3}-M_{4}-N_{1}-N_{2}-N_{3}-N_{4}}{32 \pi R A B C} \\
& =(-R+A+B+C) / 16 \pi R A B C .
\end{aligned}
$$

The calculated values of $W_{3}(R)$ for all four ranges for each case are given in Table II.

\section{Four Random Steps}

For the process involving four random steps there exist eight $M_{j}$ and eight $N_{j}$ terms. Calling $h_{1}=A, l_{2}=B$, $l_{3}=C$, and $l_{4}=D$, and assigning $A>B>C>D$, there exist 14 different complete solutions depending on the particular numerical values for $A, B, C$, and $D$. These 14 cases are listed in Table III.

For each of these cases the range of $W_{4}(R)$ is divided into eight segments. In many instances, certain solutions of $W_{4}(R)$ are common to more than one of the above cases. The complete solutions of $W_{4}(R)$ for all 14 possible cases are given in Table IV. 